that very few institutions of higher learning are giving their students anything but the most cursory advice in laboratory safety and accident prevention on the time honoured basis that God, somehow, will couple the shorn lamb with the careless chemist. Industry, whose managers tend to be better informed on the hard realities of economic loss and the trade unions' inclination to fight compensation suits, is understandably more advanced.

In this situation it is always pleasant to welcome a new book which advocates more thought in the design and manning procedures of laboratories. Such publications come in two types: one, for example the Handbook of Laboratory Hazards, now published by The Chemical Society, lays emphasis on what to do once the accident has occurred and notes those chemicals with which potential danger is always present. The volume under review, produced by the American Manufacturing Chemists Association, takes a step back and considers safety as a logical outcome of sensible design and behaviour. Except in the most general sense it is not suitable as a quick reference volume in moments of stress: although it abounds in tables, so it does in cross references which take some time to follow through. But as background reading it is most valuable, for it takes nothing for granted and leads the teacher or laboratory supervisor step by well illustrated step through all the pitfalls of space, instruments and chemicalswhen did you last test your fire extinguishers?

Frequently American practice differs from the British and there are variations in detail in regulations and materials standards: hence reference to ASTMS. Nevertheless those whose work involves the manipulation of chemicals and the safety of the people concerned will derive great benefit from keeping Guide for Safety on their bookshelves, especially in the unlikely event that a fit of generosity from UGC, DES or head office allows them to translate dreams of a new laboratory into reality.

$$
\text { Peter Farago }
$$

\section{Particle Physics}

Elementary Processes at High Energy. ("Ettore Majorana" International Centre for Scientific Culture, 1970 International School of Subnuclear Physics, a NATO-MRI-MRST Advanced Study Institute sponsored by the Regional Sicilian Government and the Weizmann Institute of Science, Erice, July 1-19.) Edited by A. Zichichi. Part A. Pp. xix +1-448. Part B. Pp. xvii +449861. (Academic: New York and London, April 1972.) $\$ 29.50$ each part.

THIS two-volume record of the 1970 International School of Subnuclear
Physics held at the hill-top town of Erice in Sicily, is a welcome addition to the scientific literature on elementary particle physics. It maintains a very high standard, and contains many lectures and discussions with permanent value.

Volume A contains the lecture courses and the review lectures, the relevant discussions being collected to follow each set. The lecture courses were of course quite topical. The opening lecture, "Electron-positron annihilation", by N. Cabibbo, was especially timely, in view of the recent operation of the electron-positron storage ring ADONE at Frascati and the early experimental results reported at the School by Italian groups working with ADONE.

G. Veneziano contributed an outstanding set of lectures about narrow resonance models compatible with duality. Besides his illuminating discussion of the origination of the model, he described clearly its generalisation to the $\mathrm{N}$-point functions which many authors have discussed for scalar particles in the literature. T. D. Lee lectured on the Lee-Wick formulation of quantum electrodynamics, which makes use of an indefinite metric; his lectures included a critical analysis of the notion of causality, as used in elementary particle physics. H. Harari presented the qualitatively successful picture of hadron reaction processes which has resulted from an amalgamation of the duality model, the absorption model, and the quark model. Here his lectures are compressed into a novel but effective format, a picture book approach consisting of twenty-nine figures, self-explanatory to some extent but each with a figure caption, and a number of remarks added by way of introduction and conclusion. Whereas Harari's "picture book" occupies twenty-two pages, the discussion following them goes on for a further thirtyfour pages.

Two review lectures on gravitational topics were given by W. Thirring from an elementary particle physicist's viewpoint, "more than a sort of exercise in Riemann geometry for esoteric theoreticians; something more down to earth". His second lectire "A trip behind the Schwarzschild radius", would, perhaps, be entitled today "Inside a black hole". Once considered outside elementary particle physics, gravitational topics are now attracting and intriguing many people in this field. Two review lectures were also contributed by T. D. Lee, the first an interesting introduction to symmetry principles in physics, and the second a critical discussion of published derivations of the $\Delta I=1 / 2$ rule from soft pion theorems. Coming from Frascati, G. Salvini surveyed the status of experiments on electron-posi- tron storage rings which bear on quantum electrodynamics, on the vector dominance model for photon interactions and on searches for new vector mesons. Finally, B. Maglic reviewed the problems of meson spectroscopy, including his proposal to study $\pi-\pi$ and $\mu-\mu$ collisions at low energy, using the self-colliding orbits of his "Precetron" device.

The seminar talks are collected together in volume B, and cover a wide range of topics, not all of which can be mentioned here. J. Adams recounted the possibilities considered for the super-CERN accelerator. His words are interesting to read today, and make us realise how wise was the final decision, in relation to the boundary conditions which existed at that time. V. Weisskopf contributed an instructive seminar about nucleon structure, as seen in the light of the scaling law now established for inelastic scattering. The study of $\pi \pi, \mathbf{K} \pi$ and $\mathbf{K} \overline{\mathbf{K}}$ interactions was covered thoroughly in an extensive series of seminars by $P$. Schlein. Especially appropriate at this School were the seminars contributed by M. Conversi and by A. Zichichi, concerning the work in progress by their groups at ADONE.

Many other seminars with much topical interest were also given, about the light-cone behaviour in quantum field theory, and its application to lepton-pair production, about vector meson dominance and deep inelastic electron-proton scattering, about the Pomeranchuk theorem and the possible ways in which it may fail, about SU(4) symmetry and its merits with respect to weak and strong interactions, and about PCAC, among other topics. One topic unusual for such a school was that discussed by $U$. Amaldi, the developments in nuclear physics which have been achieved by using high-energy particles produced by energetic accelerators. He described particularly how the analysis of quasi-free collisions, processes of the type $(\mathrm{p}, 2 \mathrm{p})$, has provided our strongest evidence for the validity of shell-model wave functions for light nuclei. The closing lecture, an interesting and authoritative history of the development of our knowledge of the properties of the weak decay interactions, was given by T. D. Lee.

The extensive discussion after each lecture has been an outstanding feature of the Erice School, and this has been recorded in full in these volumes. The audience included many students at the postdoctoral level, as well as many senior visitors who were there to lecture or to give seminars. Consequently, this discussion was at quite a high level, and frequently very illuminating. The record of these discussions is one of the most valuable aspects of this book.

R. H. Dalitz 